\title{
LA PROYECCIÓN KHARBARELLI: CÓMO Y POR QUÉ FUNCIONA ESTA TÉCNICA
}

Steve ScotT

\begin{abstract}
Resumen
La técnica de proyección conocida como Proyección Kharbarelli en el mundo del judo competitivo es un buen ejemplo de cómo diferentes culturas y sus tradiciones de lucha cuerpo a cuerpo han cambiado la fisonomía de este deporte olímpico. Esta presentación de la Proyección Kharbarelli muestra y explica la teoría, mecánica y aplicación de esta poco ortodoxa, atrayente y efectiva técnica de proyección. El autor tiene una amplia experiencia en sambo y judo y estudió esta técnica durante varios años tras ver su utilización en eventos internacionales de judo y sambo. La Proyección Kharbarelli, a pesar de considerarse "no ortodoxa" y no tener origen japonés, es efectiva porque utiliza los elementos comunes de aprovechamiento del movimiento y del equilibrio del oponente de la misma forma que lo hacen las proyecciones "tradicionales" de judo.
\end{abstract}

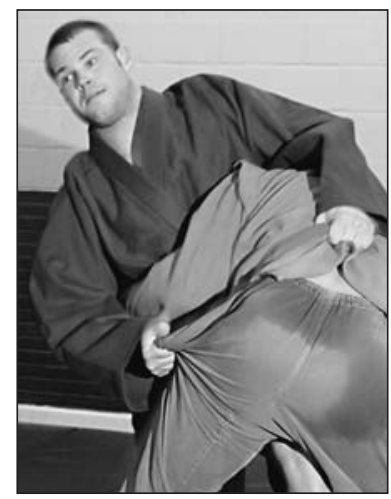

profesor Jigoro Kano.

Entre los muchos atletas superdotados que produjeron los soviéticos, destaca uno en particular por sus habilidades de proyección, únicas y muy innovadoras. Este atleta es Shota Kharbarelli, quien entre otras grandes victorias internacionales ganó la medalla de oro en la categoría de $78 \mathrm{~kg}$. en los JJ.OO. de Moscú 1980. Pero lo que le hizo famoso no fue el hecho de ganar sino cómo obtuvo sus victorias.

Kharballeri derribaba a sus oponentes con técnicas nunca antes vistas, y lo hacía con consistencia. No es casualidad que cuando este hombre lanzaba a un rival por los aires lo hiciera con una técnica calculada y bien entrenada. Cualquiera se daba cuenta de que si quería ser competitivo en judo a nivel internacional debería empezar a aprender cómo copiar y defenderse de esas inusuales proyecciones que hacían Kharbarelli y los otros soviéticos. Los puristas del judo quedaban boquiabiertos por el hecho constatado de que estas técnicas de proyección funcionasen, pero con el tiempo la Federación Internacional de Judo reconoció la técnica y la bautizó con el nombre de la persona que la hizo famosa. 
Shota Kharbarelli es Georgiano y actualmente entrena al equipo nacional de judo de Georgia. Su formación (junto con el judo) es el sambo, la forma híbrida de lucha soviética deporte de lucha/arte marcial, y en el estilo de lucha tradicional Georgiano llamado Chidaoba. Para apreciar del mejor modo posible por qué y cómo funciona la proyección "voladora" Kharbarelli, es preferible volver la vista hacia la teoría y aplicación del enfoque poco ortodoxo de los Soviéticos en el agarre de la chaqueta y en el control de la postura del oponente, y cómo esto influyó en el sambo y, en última instancia, en el judo internacional.

Muchos de los mejores atletas soviéticos de judo, sambo y lucha vienen de la antiguamente Republica Soviética de Georgia. Una de las formas más populares de lucha tradicional en Georgia es el deporte del Chidaoba, en el cual los luchadores llevan una chaqueta atada sin mangas. En este deporte el énfasis se sitúa en las proyecciones, y es popular en Georgia. El hecho de que los luchadores de Chidaoba no tengan mangas que agarrar influyó en cómo los soviéticos desarrollaron sus agresivos e innovadores agarres y la lucha por el agarre en el deporte híbrido del sambo. El énfasis del sambo en agarrar el cinturón y las solapas, llegando a la espalda del rival para aferrar la parte posterior de su chaqueta o el cinturón, y utilizar estas técnicas de agarre para romper la postura y el equilibrio del oponente, es lo que hace que funcionen las técnicas de proyección como la Proyección Kharbarelli. Básicamente, la Proyección Kharbarelli empieza con el modo en que agarras a tu oponente y rompes su postura, para luego proceder con una forma particular de control del cuerpo del oponente

Intentando reproducir el efecto de la Proyección Kharbarelli, muchos judokas han intentado imitar todos los pasos de la proyección. Este enfoque solo hace que la realización (y aprendizaje) de la técnica sea más difícil. Hay que entender que el mundo vio esta proyección a través de la lente del cámara que capturó brillantemente la proyección en acción. Muestra la proyección realizada durante un combate internacional de judo, y deberíamos darnos cuenta de que los factores fundamentales subyacentes que hacen que esta proyección funcione no se explican simplemente a través de unas fotografías. Cuando examinamos la Proyección Kharbarelli, parece que Kharbarelli trata de hacer una Gran Siega por el Interior (O Uchi Gari) y entonces cambia la dirección y levanta a su oponente por el cinturón y lo derriba de un modo espectacular. Pero lo que da inicio a todo este movimiento es el modo en que Kharbarelli agarra a su oponente. Menciono esto porque podría ser muy difícil, sino imposible, el copiar los movimientos exactos de cualquier proyección de judo o de cualquier habilidad motriz basándose en lo que aparece en una fotografía o incluso en una imagen en movimiento. Lo que estoy diciendo es que si conoces los detalles de cómo funciona la proyección, entonces serás capaz de realizarla usando tu propia capacidad física y mental, haciendo que te funcione.

El "por qué" de cómo funciona esta técnica proviene del enfoque que tiene el sambo del aprendizaje de las habilidades. Es importante entender que los luchadores de sambo no se preocupan de lo bonita que pueda parecer una técnica, sino que están más interesados en el hecho de que ésta pueda funcionar en situaciones reales con un ratio de éxito alto y consistente. Una frase que se oye habitualmente es "Haz que tu técnica te funcione". Esto fue precisamente lo que hizo Kharbarelli con la proyección que le hizo famoso. Adicionalmente, el agarre y la lucha por el agarre son elementos vitales para el éxito en el sambo. Antes de que puedas romper exitosamente la postura y el equilibrio de tu oponente para derribarlo, debes controlarlo a través del modo en que agarras su cuerpo, chaqueta o uniforme. Esta proyección en concreto, así como el resto de las proyecciones, dependen de tu agarre.

Lo que hizo Kharbarelli a su oponente finlandés en la fotografía que le hizo famoso fue realizar un ataque inicial con lo que en judo se denomina Gran Siega Interior desde un control del cinturón de su oponente por encima del hombro. Siempre he oído que este agarre al cinturón por encima del hombro que se usa en el sambo se describe como "agarre envolvente", y por ello lo llamaré así. Posiblemente al darse cuenta de que este ataque inicial encontraba la resistencia de la postura defensiva del finlandés, Kharbarelli mantuvo el control con el agarre y utilizó un levantamiento con la pierna para proyectar al oponente con un resultado espectacular. 


\section{LA PROYECCIÓN KHARBARELLI}

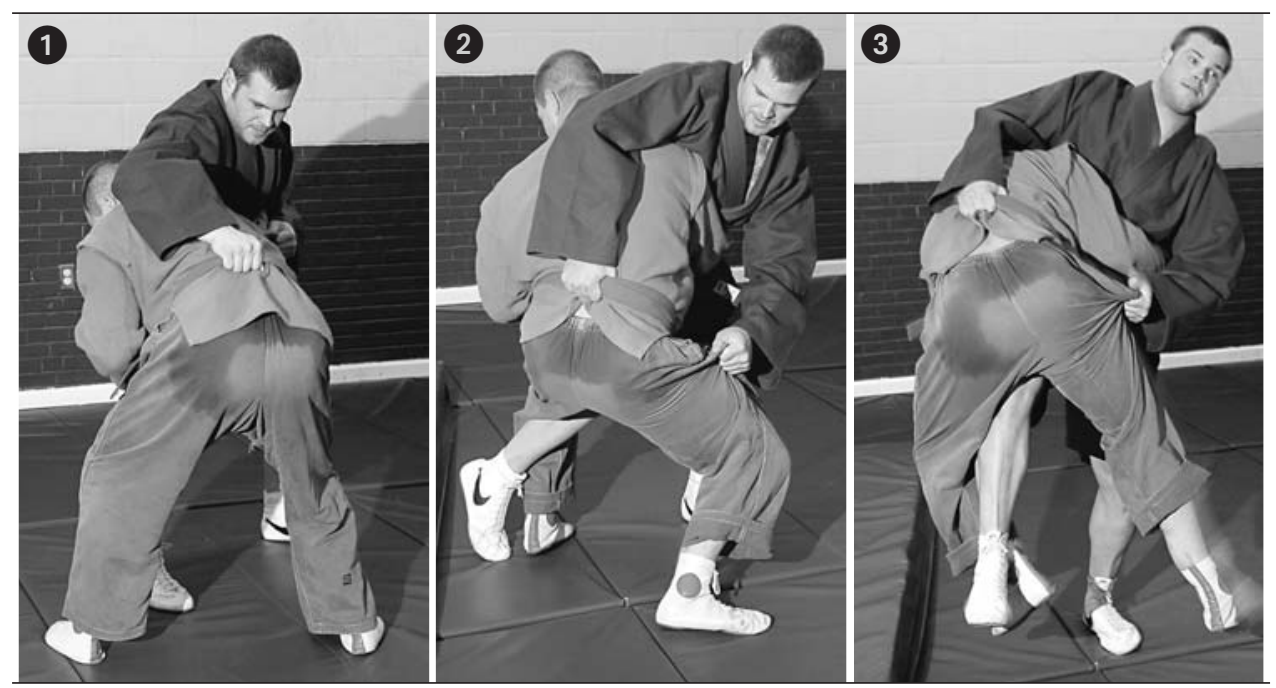

$(1)$ Trevor tiene el agarre envolvente sobre Bryan. En este caso, puedes ver (como hizo Kharbarelli), que Trevor está agarrando el cinturón y rompiendo la postura de Bryan empleando su codo para empujarle hacía abajo. El uso del codo es importante para agarrar y controlar el movimiento del oponente. Trevor puede literalmente "dirigir" y controlar el cuerpo de Bryan utilizando la presión de su codo. Trevor está usando su mano izquierda para agarrar la manga de Bryan cerca del tríceps, y hace todo lo posible para acercar a Bryan mientras empieza a atacar con O Uchi Gari. La posición de Trevor es también importante. Su pie derecho está adelantado y se coloca inmediatamente por dentro del pie izquierdo de Bryan. Esta posición inicial ayudará a cerrar rápidamente el espacio entre el cuerpo de Trevor y el de Bryan cuando Trevor inicie el ataque para proyectar.

2 Trevor ha empezado su ataque conduciendo su cuerpo (dirigiendo su cadera derecha y pie derecho) y utilizando su pierna derecha para enganchar por dentro la pierna izquierda de Bryan como muestra la fotografía. En este momento, Trevor emplea su mano izquierda para agarrar la pernera derecha de Bryan a media altura. Trevor tiene un fuerte control con su mano derecha sobre el cinturón y la espalda de Bryan. Un aspecto importante de este derribo es que Trevor se ha asegurado de que el hombro derecho de Bryan está tocando la axila derecha de Trevor. Trevor siente el hombro derecho de Bryan bajo su brazo y esto le muestra que está lo suficientemente cerca y pegado a Bryan como para enganchar su pierna o empezar a levantar su pierna. Trevor puede continuar la entrada para derribar a Bryan con un O Uchi Gari, pero en este caso Bryan ofrece suficiente resistencia y ha bajado su postura mediante una flexión de rodillas en un intento de parar el derribo.

3 Trevor siente la resistencia de Bryan y entra rápidamente con su pierna izquierda entre las piernas de Bryan como muestra la fotografía. Según hace esto, Trevor gira su cadera derecha ligeramente hacia su izquierda y usa la parte superior de su pierna derecha para despegar a Bryan de la colchoneta. Mientras Trevor hace esto, baja levemente su cuerpo doblando su pierna izquierda (pierna de apoyo). La pierna derecha de Trevor está doblada y la utiliza del mismo modo que lo haría para ayudarse cuando levanta un objeto pesado. Trevor emplea su mano derecha para levantar y utiliza su codo derecho, llevándolo hacia atrás (dirección hacia la que le proyectará), para conducir a Bryan sobre su lado derecho. Observa asimismo que Trevor está haciendo un buen uso de su mano izquierda agarrando la pernera derecha de Bryan. Trevor emplea su mano izquierda para levantar la pernera derecha de Bryan y esto le ayuda en la aplicación de la proyección.

4 Ahora Trevor está proyectando a Bryan y puedes ver cómo utiliza su pierna derecha para levantar el cuerpo de Bryan. Trevor usa su mano derecha para tirar del cinturón de Bryan mientras proyecta a Bryan por encima de su hombro izquierdo. El codo derecho de Trevor de hecho apunta hacia la dirección donde caerá Bryan. Trevor lleva su codo derecho en esa dirección y conduce a Bryan hacia donde quiere. Es

La proyección Kharbarelli: cómo y por qué funciona esta técnica $\diamond$ Steve Scott 
importante señalar que esta proyección es extremadamente explosiva y deberás hacerla con un compromiso absoluto. Trevor ha cerrado con éxito todo el espacio corporal entre él y Bryan, y ambos cuerpos estarán pegados mientras Trevor proyecta a Bryan.

5 En esta fotografía, el pie izquierdo de Trevor aún está apoyado en la colchoneta. En algunos casos (y como Kharbarelli hizo con su oponente finlandés), Trevor puede realizar un gesto tan explosivo en la proyección que él también vuela sobre la colchoneta. Sin embargo, puedes ver cómo Bryan está completamente en el aire con Trevor controlándole. Bryan está siendo proyectado por encima de su hombro izquierdo y sobre la cadera derecha de Trevor y hacia la parte derecha de su espalda. Trevor continuará girando sobre su parte posterior derecha mientras proyecta a Bryan.

6 Debido al compromiso total que Trevor ha puesto en el ataque, él girará sobre la parte derecha de su espalda mientras proyecta a Bryan por encima del hombro izquierdo de Bryan, y aterriza sobre él. Este compromiso total en la proyección es necesario para que esta funcione. Una vez que tu cuerpo está en movimiento debes seguir hasta alcanzar completamente el resultado que quieres, que es proyectar a tu oponente sobre la colchoneta.

Mucha gente en la colectividad del judo tiene etiquetado a este "judo fuerza" por los agarres y controles posturales no ortodoxos que emplea, así como por el hecho obvio de que el que proyecta está levantando y despegando a su oponente de la colchoneta para luego arrojarlo duramente sobre ella. A pesar de ello, si estudias esta técnica encontrarás que es una habilidad técnicamente convincente en la que se manifiesta la mecánica de cualquier proyección (ya venga ésta de la tradición del judo o del sambo). Con cualquier técnica, especialmente con las proyecciones, es importante que el practicante esté en forma físicamente y lo suficientemente fuerte para hacer que funcione con un oponente diestro, resistente y cualificado. Ningún atleta, en cualquier nivel de competición, pero especialmente en niveles de élite, tendrá éxito a menos que esté lo suficientemente en forma para hacer que le funcione frente a un oponente que se le resiste.

Esta proyección es indicativa de cómo el judo ha llegado a ser un auténtico deporte internacional. Después de su introducción en el escenario mundial y su inclusión en los Juegos Olímpicos, fue inevitable
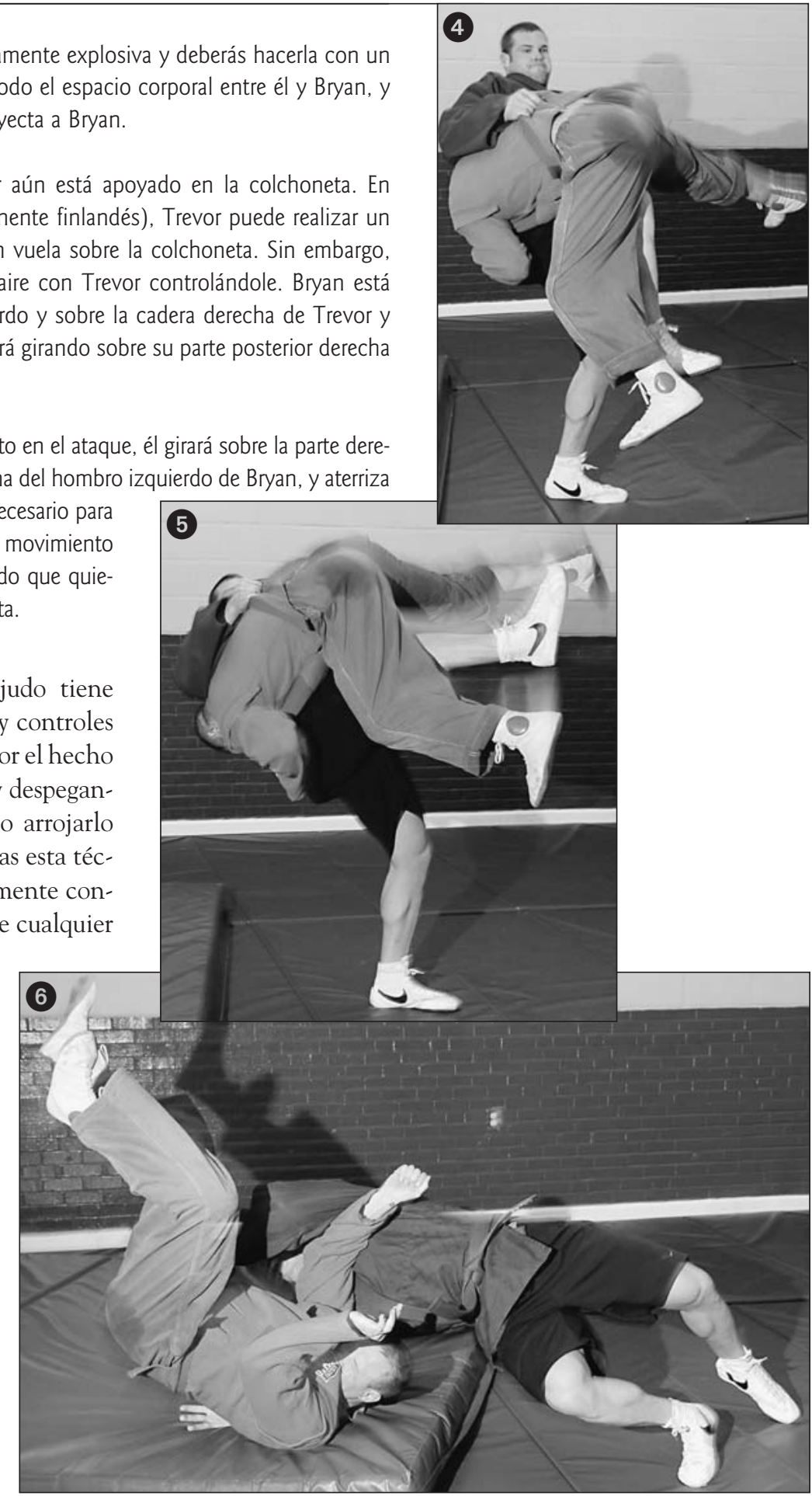
que el judo Kodokan que fundó el profesor

Kano llegase a ser una actividad competitiva basada en los resultados. Del mismo modo que han cambiado todos los eventos deportivos a lo largo de los años, el judo se ha transformado desde sus raíces en el Kodokan a ser parte de un mundo más amplio. La Proyección Kharbarelli es el resultado de un agarre agresivo, del control de la postura del oponente, y de un movimiento rápido y explosivo totalmente entregado para proyectar al rival, manifestándose todas las características del rápido y agresivo deporte en el que se ha convertido el judo. Para muchos en el mundo del judo ésta no es una bonita técnica para ver, pero, sin embargo, la belleza está en los ojos de quien mira. 